\title{
Prospective evaluation of stapled haemorrhoidopexy versus transanal haemorrhoidal dearterialisation for stage II and III haemorrhoids: three-year outcomes
}

\author{
P. Giordano - P. Nastro • A. Davies • \\ G. Gravante
}

Received: 29 October 2010/ Accepted: 13 December 2010/Published online: 12 February 2011

(C) The Author(s) 2011. This article is published with open access at Springerlink.com

\begin{abstract}
Introduction The aim of the study was to compare shortand medium-term outcomes of transanal haemorrhoidal dearterialisation (THD) versus stapled haemorrhoidopexy (SH) for the treatment of second- and third-degree haemorrhoids.

Methods Patients with second- or third-degree haemorrhoids who failed conservative treatment were randomly allocated to THD or SH. Preoperative and postoperative symptoms, postoperative pain, time until return to normal activities, complications, patient satisfaction and recurrence rates were all assessed prospectively. Patients were followed up at 2, 8 months and when the study was completed.

Results Twenty-eight patients (43\% third degree) underwent THD and 24 (38\% third degree) underwent SH. There were no significant differences in terms of postoperative pain, expected pain and analgesia requirements, but more THD patients returned to work within 4 days $(P<0.05)$. One THD patient developed a sub-mucosal haematoma after surgery, one SH patient occlusion of the rectal lumen and two rectal bleeding. At 8-month follow-up, two $\mathrm{SH}$ patients complained of faecal urgency. At 38-month follow-up (range 33-48 months), all short-term complications resolved. Patient satisfaction ("excellent/good outcome",
\end{abstract}

Paper presented as a poster at the Association of Surgeons of Great Britain and Ireland 2009, Association of Coloproctology of Great Britain and Ireland 2009 and the European Society of Coloproctology 2009.

P. Giordano $(\bowtie) \cdot$ P. Nastro · A. Davies · G. Gravante Department of Colorectal Surgery, Whipps Cross University Hospital, Whipps Cross Road, London E11 1NR, UK e-mail: pasquale.giordano@whippsx.nhs.uk
THD 89 vs. SH 87\%) and recurrence rate (THD 14 vs. SH $13 \%$ ) were similar in the two groups.

Conclusions Short-term results although similar seem to suggest SH may result in increased morbidity while return to work is quicker after THD. Medium-term results demonstrate that THD and SH have similar effectiveness.

Keywords Haemorrhoids - Stapled haemorrhoidopexy · Procedure for prolapse and haemorrhoids · Transanal haemorrhoidal dearterialisation

\section{Introduction}

Conventional haemorrhoidectomy $(\mathrm{CH})$ is the most widely used surgical procedure for the treatment of symptomatic haemorrhoids and is still considered by many surgeons as the gold-standard technique. It is very effective, relatively safe and economic, but also notoriously painful and potentially affects the mechanism of anal continence [1]. Over the years, alternative minimally invasive techniques have been developed including stapled haemorrhoidopexy (SH), also known as procedure for prolapse and haemorrhoids (PPH), and transanal haemorrhoidal dearterialisation (THD) also known as Doppler-guided haemorrhoidal artery ligation (DGHAL). SH consists of a transanal stapled circumferential rectal mucosectomy. The procedure aims to lift up the mucosa and restore the normal anatomy and physiology of the diseased haemorrhoidal tissue. Results show that the procedure is less painful and facilitates an earlier return to normal activities than to $\mathrm{CH}$ [2-5]. However, data also suggest a higher recurrence rate [6] and a small risk of serious complications [7-12]. THD is a technique that closes, under Doppler guidance, the distal branches of the superior rectal artery (SRA), thereby 
reducing the blood flow to the haemorrhoidal plexus [13]. The closure of the vessels is achieved with a dedicated proctoscope that incorporates a Doppler probe. Early data suggest a favourable comparison with $\mathrm{CH}$ in terms of postoperative pain and return to normal activities. Relapse rates seem similar [14], but long-term results are scarce $[15,16]$.

This prospective trial aims to compare short- and medium-term results of these two new treatment modalities for haemorrhoids.

\section{Materials and methods}

All patients with symptomatic second- and third-degree haemorrhoidal disease that failed a conservative treatment were offered surgical intervention in the form of THD or SH. Patients were given an explanation of the details of both procedures and were invited to participate to the study. Although there was no formal randomisation, those that agreed to participate were casually allocated to either procedure without any specific criteria for selecting one method over the other. The surgical procedure was decided blindly by the operating surgeon on the day of surgery just before examining the patient under anaesthesia and without being aware of the patient's symptoms.

All patients with fourth-degree haemorrhoids and those with a large external component were excluded and offered $\mathrm{CH}$. Furthermore, patients with complicated haemorrhoidal disease, other concomitant anal conditions, under anticoagulation therapy, with coagulation disorders or those who opted for one of the two procedures thereby refusing random allocation were also excluded.

Surgical procedure and technique

All operations were performed as a day-case procedure under general anaesthesia by the senior author (PG) or under his direct supervision by another member of the team specifically trained in the procedure. All patients were prescribed a phosphate enema prior to surgery. Both $\mathrm{SH}$ and THD procedures were carried out with the patient in the lithotomy position. THD was performed using a specifically designed proctoscope (THD PS02, THD Lab ${ }^{\mathrm{TM}}$, Correggio, Italy), which incorporates a side-sensing Doppler probe and a window beyond this for suture placement. The Doppler ultrasound transducer was used to identify the haemorrhoidal arteries at about $4 \mathrm{~cm}$ above the dentate line. Once identified, the haemorrhoidal arteries were transfixed and ligated using $2 / 0$ absorbable Vicryl ${ }^{\mathrm{TM}}$ sutures in a figure-of-eight stitch. In addition to that, a mucosopexy was performed at the same time using the same stitch starting from the level of the ligation and proceeding distally towards the dentate line, incorporating the mucosa and submucosa. The suture was stopped at about $5 \mathrm{~mm}$ from the dentate line taking care not to catch the anal mucosa in order to avoid postoperative pain.

Stapled haemorrhoidopexy was performed according to the technique described by Longo [12] using a $2 / 0$ polypropylene purse-string suture applied $4 \mathrm{~cm}$ above the dentate line including mucosa and submucosa. The dedicated circular stapling device (PPH 03, Ethicon EndoSurgery ${ }^{\mathrm{TM}}$, Ohio, USA) was then used for mucosectomy and anopexy. The excised specimen of the SH group was inspected and sent for histological examination. Finally, an absorbable gelatine sponge dressing was placed in the anal canal of all patients.

Anaesthesia and operative time were recorded in a computerised log. Patients were discharged with rectal $2 \%$ lignocaine gel, oral diclofenac (50 mg tid) and co-dydramol (paracetamol $1000 \mathrm{mg}$-dihydrocodeine $60 \mathrm{mg}$ qid) to be used as required for 10 days. Patients also received regular laxatives for 2 weeks (Hyspagula sachet po bid and Lactulose $10 \mathrm{ml}$ po bid).

\section{Assessment and postoperative follow-up}

All data were prospectively collected. Data included patients' demographics and relevant history. The degree of severity of haemorrhoidal symptoms was scored for each patient using a specifically designed questionnaire assessing 5 different parameters, each scoring from 0 to 4 with 0 corresponding to no symptoms at all and 4 to the presence of the symptoms on a daily basis or with every bowel movement (Table 1). A total score of 0 corresponded to the complete absence of haemorrhoidal symptoms, while a total score of 20 corresponded to the worst possible degree of symptoms (Table 1). Postoperative pain was assessed using a standardised visual analogue score $0-10(0=$ no pain, $10=$ the worse possible pain) with patients asked to record the most severe episode. Patients were also asked to record in a similar manner the expected pain from -5 to +5 VAS, with -5 corresponding to the actual pain being much better than expected, 0 as expected and +5 much worse than expected.

Patients were reviewed in the outpatient clinic at 8 weeks and 8 months and reassessed with a telephone interview at 3 years. During the interview, the questionnaire on symptoms was completed again and unless patients were completely asymptomatic they were recalled and evaluated in the outpatient clinic. Patient satisfaction was assessed at 3 years with 4 categories: excellent, good, fair and poor. 
Table 1 Symptom questionnaire

QoL quality of life

\section{Statistical analysis}

All collected data were entered into an Excel database (Microsoft Corporation, Redmond-Washington, USA) and analysed with the Statistical Package for the Social Sciences Windows version 13.0 (SPSS, Chicago, Illinois, USA). Descriptive statistics for quantitative continuous variables were the mean and standard deviation after confirmation of normal distribution, otherwise median and range. Descriptive statistics for qualitative categorical variables were performed using frequencies. Comparison of groups (SH vs. THD) was performed with Student's $t$-test for continuous parametric, the Mann-Whitney test for continuous non-parametric and the chi-square test or Fisher's exact test for categorical variables (Fisher's if counts were inferior to 5). A $P$ value of $<0.05$ was considered statistically significant.

\section{Results}

Between September 2004 and December 2005, 64 consecutive patients were evaluated. Twelve patients were excluded from this group (Fig. 1), leaving 52 patients for analysis. Demographic and preoperative clinical data are summarised in Table 2. THD and SH groups were homogeneous for age, sex, previous haemorrhoidal surgery, degree of prolapse and preoperative symptom score. All patients had previously received at least one injection of sclerotherapy, 7 had undergone haemorrhoidal banding.

\section{Early results}

Early postoperative results are summarised in Table 3. No differences were observed for the operative time. There was a trend towards less pain in the THD group although this did not reach statistical significance. All patients but one in both groups were discharged on the same day they had surgery. There was a significant difference between the groups in terms of return to work that favoured the THD group (Table 3).

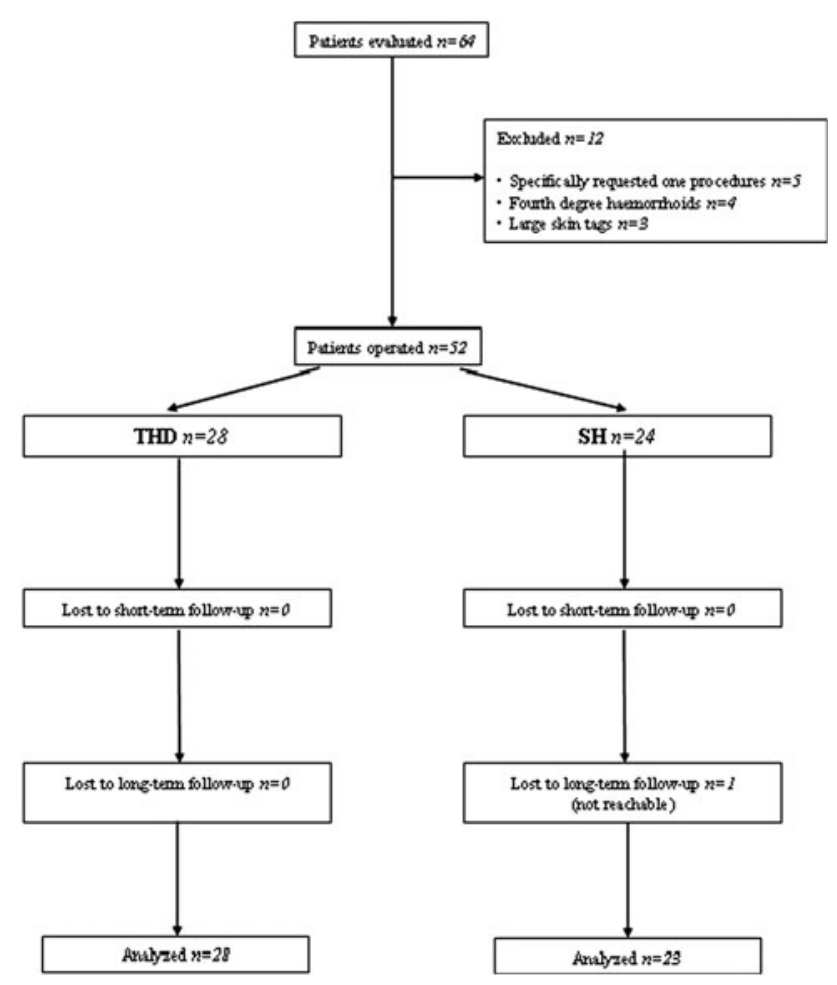

Fig. 1 Flow diagram of patients at each stage of treatment $T H D$ transanal haemorrhoidal dearterialisation, $S H$ stapled haemorrhoidopexy

No significant differences were observed for the rate of postoperative surgical complications or readmissions (Tables 3, 4). In 2 THD patients, the tip of the needle snapped off during ligation and was not retrieved. This did not cause any symptoms and both patients had a successful outcome. Another patient in the THD group experienced urinary retention. He had to be catheterised overnight and was discharged the following day. One patient in the $\mathrm{SH}$ group developed complete occlusion of the rectal lumen following firing of the stapler. The complication was successfully managed endoscopically, and the patient was discharged home 2 days later. Again, even in this case, the haemorrhoidal symptoms resolved successfully after surgery [17]. In the SH group, 2 patients with postoperative bleeding had to be readmitted and managed conservatively. 
Table 2 Demographic data

$M$ males, $F$ females, $\mathrm{CH}$ conventional haemorrhoidectomy, THD transanal haemorrhoidal dearterialisation, $\mathrm{SH}$ stapled haemorrhoidopexy

\begin{tabular}{llll}
\hline & THD $(n=28 ; 54 \%)$ & SH $(n=24 ; 46 \%)$ & $P$ \\
\hline Age (years) & $54(23-73)$ & $48(35-78)$ & NS \\
Sex (males/females) & $20 \mathrm{M} / 8 \mathrm{~F}$ & $16 \mathrm{M} / 8 \mathrm{~F}$ & NS \\
Previous CH & $1(4 \%)$ & $0(0 \%)$ & NS \\
Haemorrhoidal degree & & & \\
Second degree & $16(57 \%)$ & $15(62 \%)$ & NS \\
$\quad$ Third degree & $12(43 \%)$ & $9(38 \%)$ & NS \\
Preoperative scoring system & $13.6(8-20)$ & $13.3(9-18)$ & NS \\
\hline
\end{tabular}

Table 3 Early postoperative results

\begin{tabular}{|c|c|c|c|}
\hline & THD $(n=28 ; 54 \%)$ & $\mathrm{SH}(n=24 ; 46 \%)$ & $P$ \\
\hline Operating time (min) & $30(20-45)$ & $33(18-100)$ & NS \\
\hline Postoperative pain (VAS) & $2(0-9)$ & $3.5(1-10)$ & NS \\
\hline Postoperative versus expected pain & $-2(-5,1)$ & $-3(-5,2)$ & NS \\
\hline Delayed discharge & $1(4 \%)$ & $1(4 \%)$ & NS \\
\hline Time required to return to normal activities (days) & $3.2(1-11)$ & $6.3(4-14)$ & $<0.01$ \\
\hline Patients with return to work or normal activities at postoperative day 4 & $25(89 \%)$ & $12(50 \%)$ & $<0.05$ \\
\hline Readmissions & $0(0 \%)$ & $3(12 \%)$ & NS \\
\hline
\end{tabular}

$T H D$ transanal haemorrhoidal dearterialisation, $S H$ stapled haemorrhoidopexy

None of them required blood transfusions. Another patient in the $\mathrm{SH}$ group also required readmission on the second postoperative day because of severe pain (Tables 3, 4).

Three-year results

The overall median follow-up was 38 months (range 33-48 months). Only one patient in the SH group was not contactable at this time. This patient was asymptomatic when seen at 8 months.

Table 4 Postoperative complications

\begin{tabular}{llll}
\hline & $\begin{array}{l}\text { THD } \\
(n=28 ; 54 \%)\end{array}$ & $\begin{array}{l}\text { SH } \\
(n=24 ; 46 \%)\end{array}$ & $P$ \\
\hline Faecal incontinence & $0(0 \%)$ & $0(0 \%)$ & NS \\
$\begin{array}{l}\text { Transient faecal } \\
\text { urgency }\end{array}$ & $0(0 \%)$ & $2(8 \%)$ & NS \\
Submucosal haematoma & $1(4 \%)$ & $0(0 \%)$ & NS \\
Technical problems & $2(7 \%)$ & $0(0 \%)$ & NS \\
Postoperative bleeding & $0(0 \%)$ & $2(8 \%)$ & NS \\
Rectal stenosis & $0(0 \%)$ & $1(4 \%)$ & NS \\
Urinary retention & $1(4 \%)$ & $0(0 \%)$ & NS \\
Severe postoperative & $0(0 \%)$ & $1(4 \%)$ & NS \\
$\quad$ pain & & & \\
Total complications & $4(14 \%)$ & $6(25 \%)$ & NS \\
\hline
\end{tabular}

$T H D$ transanal haemorrhoidal dearterialisation, $S H$ stapled haemorrhoidopexy
Medium-term results are shown in Table 5. There was no significant difference in recurrence between the groups (14 vs. 13\%). Five patients ( 3 THDs and 2 SHs) had prolapsed haemorrhoids and successfully proceeded to $\mathrm{CH}$. Two patients, one in each group, suffering from haemorrhoidal bleeding were happy to avoid further intervention (Table 5). Two other patients suffered of occasional pruritus but had no evidence of haemorrhoidal disease on clinical evaluation.

There was no significant difference in the postoperative symptom scores between THD and SH (Table 5; $P=\mathrm{NS}$ ). Both techniques significantly reduced the scores when compared to the preoperative values $(P<0.0001)$. As both groups had also similar preoperative scores (Table 2), it appeared that THD and SH were associated with a similar degree of improvement.

No differences were observed between the groups as regards patient satisfaction. Satisfaction was excellent or good in $89 \%(n=25)$ of patients in the THD group and $87 \%(n=20)$ in the SH group $(P=\mathrm{NS})$. Those patients that rated their satisfaction as excellent were completely asymptomatic at the time of the interview (Table 5).

\section{Discussion}

The ideal surgical option for the treatment of symptomatic haemorrhoids is a technique able to provide long-term 
Table 5 Medium-term postoperative results

\begin{tabular}{lccc}
\hline & THD $(n=28 ; 55 \%)$ & SH $(n=23 ; 45 \%)$ & $P$ \\
\hline Postoperative scoring system & $1.1(0-7)$ & $1.6(0-12)$ & NS \\
Total recurrences & $4(14 \%)$ & $3(13 \%)$ & NS \\
Haemorrhoidal prolapsed & $3(11 \%)$ & $2(9 \%)$ & NS \\
Frequent bleeding & $1(4 \%)$ & $1(4 \%)$ & NS \\
Satisfaction rate & & & \\
Excellent & $19(68 \%)$ & $15(65 \%)$ & NS \\
Good & $5(18 \%)$ & $3(13 \%)$ & NS \\
Fair & $2(7 \%)$ & $2(9 \%)$ & NS \\
Poor & $2(7 \%)$ & $3(13 \%)$ & NS \\
\hline
\end{tabular}

THD transanal haemorrhoida dearterialisation, $\mathrm{SH}$ stapled haemorrhoidopexy

and lifts up the mucosa at the same time. In spite of using these methods for correcting the causes of the haemorrhoidal disease, Aigner et al. [20] recently questioned the effectiveness of $\mathrm{SH}$ in achieving the dearterialisation, showing no postinterventional changes in either arterial calibre or arterial blood flow. These findings suggest that mucosopexy may play a more important role in $\mathrm{SH}$ for the treatment of haemorrhoidal disease. The same author also demonstrated that transmural branches of the SRA enter the rectal wall very distally, below the ligation line of THD and the staple line of SH. These vessels might be responsible for the recurrences observed after THD and SH [20]. However, a mucosopexy almost down to the dentate line like the one used in our THD patients should also be able to deal with these most distal branches.

Numerous studies clearly demonstrated the short-term advantages of $\mathrm{SH}$ compared to $\mathrm{CH}$ especially in terms of postoperative pain and quicker recovery [3, 4, 21]. On the contrary, only one trial compared THD to $\mathrm{CH}$ and showed that THD requires less postoperative analgesia [14]. SH and THD are both performed above the sensitive anoderm and therefore should cause less postoperative pain than to $\mathrm{CH}$. Our results confirm that both $\mathrm{SH}$ and THD produce minimal postoperative pain with no significant differences among the techniques. Furthermore, we observed a definite advantage of THD: patients returned to work earlier. It is possible that this occurred because patients in the THD group experienced less postoperative discomfort that was however not demonstrated due to the relatively small number of patients included in the study. Indeed, it has been reported that when $\mathrm{TDH}$ is compared to $\mathrm{SH}$, postoperative pain following THD is lower during the first week [22].

The rare but definite risk of major complications after SH has been clearly documented [7], but no reports of major complications following THD are currently available [15]. We believe that the risk of major complications following SH is mainly related to the "blind" excision of the rectal wall. Since THD is a non-excisional technique, the possibility of major problems should be virtually rectal wall that transects the terminal branches of the SRA 
eliminated. In our study, the tip of the needle snapped off during the ligation in 2 THD patients. The problem was related to a defect in the batch of needles used. Indeed, this was the only time this unusual problem was encountered by the senior author of this study in more than 400 procedures performed over a 7-year period. In the SH group, postoperative pain, bleeding and one case of rectal lumen closure after firing of the stapler accounted for the increased rates of both delayed discharges and readmissions.

A recent meta-analysis of long-term outcomes of $\mathrm{SH}$ vs. $\mathrm{CH}$ demonstrated a significantly higher overall rate of prolapse recurrence in the SH group [6]. The difference was even more significant for results in patients with thirdand fourth-degree haemorrhoids. Other outcome measures including bleeding and patients satisfaction were similar in the 2 groups. The only prospective trial available comparing THD to $\mathrm{CH}$ reported a recurrence rate of $20 \%$ for THD and $16 \%$ for $\mathrm{CH}$ [14], but this study included patients with all degrees of prolapsed and the THD technique used in this study did not include a mucosopexy. A recent review of all published data on THD included almost 2000 patients and reported an overall recurrence rate of $9 \%$ for prolapse and $7.8 \%$ for bleeding [15]. When those studies with a follow-up of 1 year or more were analysed, the prolapse was present in $10.8 \%$ of patients (46/427) and bleeding in $9.7 \%$ (49/507). Individual studies reporting long-term results of THD for third-degree haemorrhoids have shown recurrence rates of $12-13.5 \%[16,23]$. The impact of the rectal plication on recurrence rates following THD has also been reported [24]. In a recently published prospective trial, Khafagy et al. [25] randomised 45 patients with haemorrhoids to $\mathrm{SH}, \mathrm{CH}$ and DGHAL. The degree of haemorrhoids included is not clear since the authors first stated that third- and fourth-degree haemorrhoids were included but then that patients with nonreducible haemorrhoids were excluded. This study demonstrated pain to be significantly worse following $\mathrm{CH}$. While there was no significant difference between the groups regarding improvement of symptoms such as bleeding and pain, prolapse completely resolved following $\mathrm{CH}$ while it improved in only 67 and $60 \%$ of patients following $\mathrm{SH}$ and DGHAL, respectively. The length of follow-up was not stated. In another prospective trial comparing THD with mucosopexy to SH for grades III and IV haemorrhoids, 78 and $83 \%(P=0.648)$ of patients had complete resolution of symptoms at 6 weeks follow-up [20]. In this study, persistent prolapse appeared to be more common following THD (11 vs. 22\%). However, more patients with fourth-degree haemorrhoids were randomised to the THD group, which may have influenced the results [26].

The results of our study confirmed recurrence rates of 14 and $13 \%$, respectively, for THD and SH at three-year follow-up, well within the range of those reported in the literature. Based on these findings, the advocates of haemorrhoidectomy might point to the higher recurrence rates of less aggressive surgery compared to $\mathrm{CH}$. However, it is important to bear in mind that the early postoperative benefits of SH and THD could easily overcome the higher incidence rate of late symptoms and make these procedures much more appealing to patients.

We acknowledge that the study has a number of limitations. Equivalence of most outcomes may reflect sample size, and there is potential for surgeon-related bias in relation to choice of procedure for each patient. Nevertheless, it is the first to assess directly these two procedures and provide a decent length of follow-up. These results show that THD and SH have similar effectiveness for the treatment of second- and third-degree haemorrhoids although THD may yield certain advantages in terms of risk of potentially serious complications, postoperative pain and time off work. Given its medium-term results comparable to $\mathrm{SH}$ in terms of recurrence rates and patient satisfaction, we suggest that THD could be considered a valid alternative first-line surgical option for the treatment of second- and third-degree haemorrhoids, although larger randomised studies are needed to better establish the definitive role of this technique.

Conflict of interest The senior author Pasquale Giordano is a trainer in the THD technique. The authors Piero Nastro, Andrew Davies and Gianpiero Gravante have no conflicts of interest or financial ties to disclose.

Open Access This article is distributed under the terms of the Creative Commons Attribution Noncommercial License which permits any noncommercial use, distribution, and reproduction in any medium, provided the original author(s) and source are credited.

\section{References}

1. Lunniss PJ, Gladman MA, Hetzer FH, Williams NS, Scott SM (2004) Risk factors in acquired faecal incontinence. J R Soc Med 97:111-116

2. Ortiz H, Marzo J, Armendariz P (2002) Randomized clinical trial of stapled haemorrhoidopexy versus conventional diathermy haemorrhoidectomy. Br J Surg 89:1376-1381

3. Shao WJ, Li GC, Zhang ZH, Yang BL, Sun GD, Chen YQ (2008) Systematic review and meta-analysis of randomized controlled trials comparing stapled haemorrhoidopexy with conventional haemorrhoidectomy. Br J Surg 95:147-160

4. Nisar PJ, Acheson AG, Neal KR, Scholefield JH (2004) Stapled hemorrhoidopexy compared with conventional hemorrhoidectomy: systematic review of randomized, controlled trials. Dis Colon Rectum 47:1837-1845

5. Pramateftakis MG (2010) The role of hemorrhoidopexy in the management of 3rd degree hemorrhoids. Tech Coloproctol 14(Suppl 1):S5-S7

6. Giordano P, Gravante G, Sorge R, Ovens L, Nastro P (2009) Long-term outcomes of stapled hemorrhoidopexy vs 
conventional hemorrhoidectomy: a meta-analysis of randomized controlled trials. Arch Surg 144:266-272

7. Pescatori M, Gagliardi G (2008) Postoperative complications after procedure for prolapsed hemorrhoids (PPH) and stapled transanal rectal resection (STARR) procedures. Tech Coloproct 12:7-19

8. Molloy RG, Kingsmore D (2000) Life threatening pelvic sepsis after stapled haemorrhoidectomy. Lancet 355:810

9. Serventi A, Rassu PC, Giaminardi E, Vitali GC, Brigli G (2010) Fecaloma in an iatrogenic diverticulum: an unusual complication of the procedure for prolapsed hemorrhoids (PPH). Tech Coloproctol 14:371-372

10. Büyükaşik O, Hasdemir OA, Cöl C (2009) Rectal lumen obliteration from stapled hemorrhoidopexy: can it be prevented? Tech Coloproctol 13:333-335

11. Khubchandani I, Fealk MH, Reed JF III (2009) Is there a postPPH syndrome? Tech Coloproctol 13:141-144

12. Lacerda-Filho A, Assunção GM, de Oliveira TA (2009) Neuropathic pain after stapled hemorrhoidopexy. Tech Coloproctol 13:255-256

13. Morinaga K, Hasuda K, Ikeda T (1995) A novel therapy for internal hemorrhoids: ligation of the hemorrhoidal artery with a newly devised instrument (Moricorn) in conjunction with a Doppler flowmeter. Am J Gastroenterol 90:610-613

14. Bursics A, Morvay K, Kupcsulik P, Flautner L (2004) Comparison of early and 1-year follow-up results of conventional hemorrhoidectomy and hemorrhoid artery ligation: a randomized study. Int J Colorectal Dis 19:176-180

15. Giordano P, Overton J, Madeddu F, Zaman S, Gravante G (2009) Transanal hemorrhoidal dearterialization: a systematic review. Dis Colon Rectum 52:1665-1671

16. Faucheron JL, Gangner Y (2008) Doppler-guided hemorrhoidal artery ligation for the treatment of symptomatic hemorrhoids: early and three-year follow-up results in 100 consecutive patients. Dis Colon Rectum 51:945-949

17. Longo A (1998) Treatment of haemorrhoidal disease by reduction of mucosa and haemorrhoidal prolapse with a circular suturing device: a new procedure. In: Monduzzi Editore (Ed.), 6th World congress of endoscopic surgery, Bologna, pp 777-784

18. Thomson WH (1975) The nature of haemorrhoids. Br J Surg $62: 542-552$

19. Lestar B, Penninckx F, Kerremans R (1989) The composition of anal basal pressure. An in vivo and in vitro study in man. Int $\mathbf{J}$ Colorectal Dis 4:118-122

20. Aigner F, Bodner G, Conrad F, Mbaka G, Kreczy A, Fritsch H (2004) The superior rectal artery and its branching pattern with regard to its clinical influence on ligation techniques for internal hemorrhoids. Am J Surg 187:102-108

21. Tjandra JJ, Chan MK (2007) Systematic review on the procedure for prolapse and hemorrhoids (stapled hemorrhoidopexy). Dis Colon Rectum 50:878-892

22. Festen S, van Hoogstraten MJ, van Geloven AA, Gerhards MF (2009) Treatment of grade III and IV haemorrhoidal disease with PPH or THD. A randomised trial on postoperative complication and short-term results. Int J Colorectal Dis 24:1401-1405

23. Scheyer M, Antonietti E, Rollinger G, Mall H, Arnold S (2006) Doppler-guided hemorrhoidal artery ligation. Am J Surg 191:89-93

24. Dal Monte PP, Tagariello C, Sarago M et al (2007) Transanal haemorrhoidal dearterialisation: nonexcisional surgery for the treatment of haemorrhoidal disease. Tech Coloproctol 11:333-338

25. Khafagy W, El Nakeeb A, Fouda E et al (2009) Conventional haemorrhoidectomy, stapled haemorrhoidectomy, Doppler guided haemorrhoidectomy artery ligation; post operative pain and anorectal manometric assessment. Hepatogastroenterology 56:1010-1015

26. Teo JY, Kam MH, Eu KW (2010) Letter to the editor on the article "Treatment of grade III and IV haemorrhoidal disease with PPH or THD. A randomized trial on postoperative complications and short-term results". Int J Colorectal Dis 25:1385 